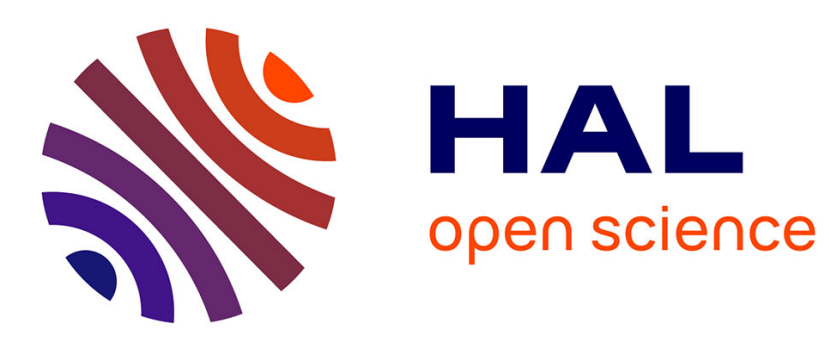

\title{
Impacts of Wetting Layer and Excited State on the Modulation Response of Quantum-Dot Lasers
}

\author{
Cheng Wang, Frederic Grillot, Jacky Even
}

\section{To cite this version:}

Cheng Wang, Frederic Grillot, Jacky Even. Impacts of Wetting Layer and Excited State on the Modulation Response of Quantum-Dot Lasers. IEEE Journal of Quantum Electronics, 2012, 48 (9), pp.1144. 10.1109/JQE.2012.2205224 . hal-00725665

\section{HAL Id: hal-00725665 \\ https://hal.science/hal-00725665}

Submitted on 27 Aug 2012

HAL is a multi-disciplinary open access archive for the deposit and dissemination of scientific research documents, whether they are published or not. The documents may come from teaching and research institutions in France or abroad, or from public or private research centers.
L'archive ouverte pluridisciplinaire HAL, est destinée au dépôt et à la diffusion de documents scientifiques de niveau recherche, publiés ou non, émanant des établissements d'enseignement et de recherche français ou étrangers, des laboratoires publics ou privés. 


\title{
Impacts of Wetting Layer and Excited State on the Modulation Response of Quantum-Dot Lasers
}

\author{
Cheng Wang, Frédéric Grillot, Senior Member, IEEE, and Jacky Even
}

\begin{abstract}
The modulation response of quantum-dot (QD) lasers is studied. Based on a set of four rate equations, a new analytical modulation transfer function is developed via a small-signal analysis. The transfer function can clearly describe the impacts of the wetting layer and the excited states: finite carrier capture and carrier relaxation times as well as the Pauli blocking limits the modulation bandwidth. The definitions of the resonance frequency and the damping factor of QD lasers are also improved. From the analysis, it is demonstrated that carrier escape from the ground state to the excited states leads to a nonzero resonance frequency at low bias powers associated to a strong damping factor.
\end{abstract}

Index Terms-Modulation response, semiconductor laser, quantum-dot (QD).

\section{INTRODUCTION}

Q UANTUM-DOT lasers have attracted lots of attention as next-generation laser sources for fiber telecommunication networks, because of the promising properties such as low threshold current [1], temperature insensitivity [2], high bandwidth [3], [4] and low chirp [5], [6]. Particularly, directly modulated lasers (DML) have been expected to play a major role in the next-generation telecommunication links for cooler-less and isolator-free applications. However, one of the major drawbacks of QD lasers concern the modulation bandwidth, which remains still limited at room temperature to about $10-12 \mathrm{GHz}$ within $1.3-1.6 \mu \mathrm{m}$ operating wavelengths [7]. In order to enhance the modulation properties several solutions have been explored including injection-locking [8], tunnelling injection [9] or p-doping [10]. However, for standard QD lasers (namely without any artificial solutions), the modulation bandwidth is still much lower than the best values reported on quantum well (QW) lasers [11]. Consequently, it is essential to clarify the origin of such a limitation. It is well known that the modulation bandwidth is strongly dependent on the resonance frequency as well as on the damping factor.

Mansucript received April 4, 2012; revised June 13, 2012; accepted June 15, 2012. Date of publication June 19, 2012; date of current version June 29, 2012. This work was supported in part by the TELDOT Project, French National Initiative, ANR, and by Rennes Metropole.

C. Wang and J. Even are with the Institut National des Sciences Appliquées, Université Européenne de Bretagne, Rennes 35708, France (e-mail: cheng.wang@insa-rennes.fr; jacky.even@insa-rennes.fr).

F. Grillot is with the Institut National des Sciences Appliquées, Université Européenne de Bretagne, Rennes 35708, France, and also with Telecom Paristech, École Nationale Supérieure des Télécommunications, Paris 75013, France (e-mail: frederic.grillot@insa-rennes.fr).

Color versions of one or more of the figures in this paper are available online at http://ieeexplore.ieee.org.

Digital Object Identifier 10.1109/JQE.2012.2205224
The resonance frequency is limited by the maximum modal gain and by gain compression effects [12] while the damping factor much stronger in QD lasers sets the limit of the modulation bandwidth [13]-[15]. Although the underlying physical origins are still under research, gain compression [14], Coulomb interaction [15] and carrier capture dynamics [16]-[18] have been proposed to explain the strong damping in QD lasers. For instance, Asryan et al. also showed that the carrier capture from the optical confinement layer into QDs can strongly limit the modulation bandwidth [19]. It is well-known that the analysis of a semiconductor laser dynamics can be conducted through the analytical expression of the transfer function coming out from the standard two rate equations [12]. However this approach, which is more appropriate to QW lasers is not always suitable for QD devices because of a different and a more sophisticated carrier dynamics. Over the last years, many theoretical studies have been devoted to investigate the carrier dynamics in QD lasers [11], [20]-[23], [35]. On the first hand, one approach relies on the use of pure numerical simulations with coupled differential rate equations [21]-[23]. Although such a method can be very powerful, it does not always give a lot of flexibility to identify the roles of the key-parameters contributing to the laser's degradation properties. On the other hand, Sugawara et. al proposed an empirical expression to fit the experimental modulation response [20] while Fiore et. al developed an indirect approach to recast the set of complex QD rate equations into the standard QW rate equations [11].

In this paper, we theoretically investigate the intensity modulation (IM) properties of InAs/InP(311B) QD lasers [24] with a cascade model including a direct relaxation channel [25]. A new analytical modulation transfer function is derived through a small-signal analysis of the differential rate equations. The analysis gives a good understanding of the impacts of the wetting layer (WL) and of the excited states (ES). It is demonstrated that finite carrier capture time, finite carrier relaxation and Pauli blocking limits the maximum bandwidth. The definitions of the resonance frequency and the damping factor are also improved. The analytical derivation points out that carrier escape from the ground state (GS) to the ES leads to a non-zero resonance frequency at low bias powers and to a strong damping factor as commonly observed in QD lasers. These theoretical investigations are of prime importance for the optimization of low cost sources for optical telecommunications as well as for a further improvement of QD laser performances. The paper is organized as follows. In section II, the numerical model describing QD carrier 
dynamics is presented. Then we derive the new modulation transfer function through a small-signal analysis of the differential rate equations. New relationships for the resonance frequency and the damping factor are also demonstrated. In section III, numerical results are presented and compared to experimental ones. Calculations are also compared to those obtained from the standard QW model. Finally, we summarize our results and conclusions in section IV.

\section{NumericAl Model Description}

The numerical model of the QD laser holds under the assumption that the active region consists of only one QD ensemble, where QDs are interconnected by a wetting layer (WL) [25]. The QD ensemble includes two energy levels: a two-fold degenerate ground state (GS) and a four-fold degenerate excited state (ES). The QDs are assumed to be always neutral, electrons and holes are treated as electron-hole (eh) pairs, which mean that the system is in excitonic energy states. Carriers are supposed to be injected directly from the contacts into the WL levels, so the carrier dynamics in the barrier is not taken into account in the model.

Fig. 1 shows the schematic of the carrier dynamics in the conduction band. Firstly, the external injected carrier fills directly into the WL reservoir, some of the carriers are then either captured into the ES within time $\tau_{E S}^{W L}$ or directly into the GS within time $\tau_{G S}^{W L}$, and some of them recombine spontaneously with a spontaneous emission time $\tau_{W L}^{s p o n}$. Once in the ES, carriers can relax into the GS within time $\tau_{G S}^{E S}$ or recombine spontaneously. On the other hand, carrier can also be thermally reemitted from the ES to the WL with an escape time $\tau_{W L}^{E S}$, which is governed by the Fermi distribution for the quasi-thermal equilibrium without external excitation [26]. Similar dynamic behavior is followed for the carrier population on the GS level with regards to the ES. Stimulated emission occurs from the GS when the threshold is reached, and that from the ES is not taken into account in the model. Following the sketch of Fig. 1, the four rate equations on carrier and photon densities are as follows:

$$
\begin{aligned}
\frac{d N_{W L}}{d t}= & \frac{I}{q V_{W}}+\frac{N_{E S}}{\tau_{W L}^{E S}} \frac{V_{D}}{V_{W}}-\frac{N_{W L}}{\tau_{E S}^{W L}} f_{E S}-\frac{N_{W L}}{\tau_{G S}^{W L}} f_{G S} \\
& -\frac{N_{W L}}{\tau_{W L}^{s p o n}}+\frac{N_{G S}}{\tau_{W L}^{G S}} \frac{V_{D}}{V_{W}} \\
\frac{d N_{E S}}{d t}= & \frac{N_{W L}}{\tau_{E S}^{W L}} \frac{V_{W}}{V_{D}} f_{E S}+\frac{N_{G S}}{\tau_{E S}^{G S}} f_{E S}-\frac{N_{E S}}{\tau_{W L}^{E S}} \\
& -\frac{N_{E S}}{\tau_{G S}^{E S}} f_{G S}-\frac{N_{E S}}{\tau_{E S}^{s p o n}} \\
\frac{d N_{G S}}{d t}= & \frac{N_{W L}}{\tau_{G S}^{W L}} \frac{V_{W}}{V_{D}} f_{G S}+\frac{N_{E S}}{\tau_{G S}^{E S}} f_{G S}-\frac{N_{G S}}{\tau_{E S}^{G S}} f_{E S}-\frac{N_{G S}}{\tau_{G S}^{\text {spon }}} \\
& -g_{G S} v_{g} S_{G S}-\frac{N_{G S}}{\tau_{W L}^{G S}} \\
\frac{d S_{G S}}{d t}= & \Gamma_{p} g_{G S} v_{g} S_{G S}-\frac{S_{G S}}{\tau_{P}}+\Gamma_{p} \beta_{S P} \frac{N_{G S}}{\tau_{G S}^{s p o n}}
\end{aligned}
$$

where $N_{W L, E S, G S}$ are carrier densities in WL, ES, GS, and $S_{G S}$ is photon density in the cavity with GS resonance

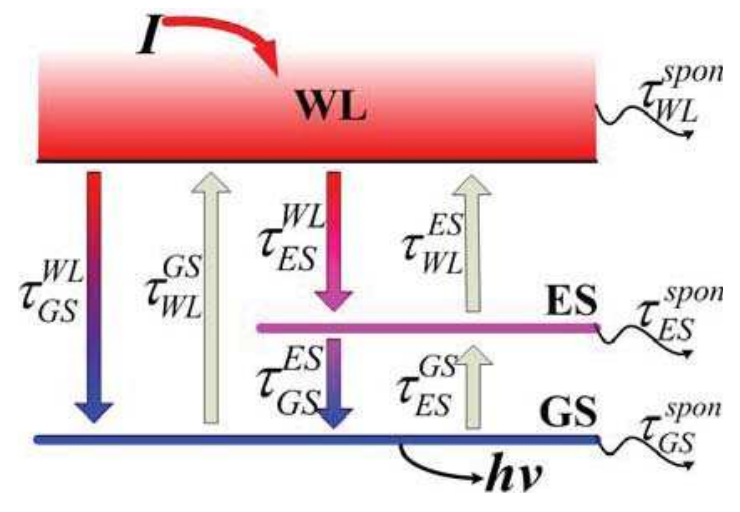

Fig. 1. Sketch of the carrier dynamics model, including a direct relaxation channel.

energy. $\beta_{S P}$ is the spontaneous emission factor, $\Gamma_{p}$ the optical confinement factor, $\tau_{p}$ the photon lifetime and $v_{g}$ the group velocity. $V_{W}$ and $V_{D}$ are the volumes of the $\mathrm{WL}$ and the QD, respectively. The GS gain is given by:

$$
g_{G S}=a_{G S} N_{B}\left(\frac{N_{G S}}{N_{B}}-1\right)
$$

where $a_{G S}$ is the differential gain and $N_{B}$ is the QD density. In what follows, it is important to stress that effects of gain compression are not taken into account. In (1)-(3), $f_{G S, E S}$ are the Pauli blocking factors of the GS and the ES, respectively, which correspond to the probabilities to find an empty carrier state:

$$
f_{G S}=1-\frac{N_{G S}}{2 N_{B}} ; \quad f_{E S}=1-\frac{N_{E S}}{4 N_{B}} .
$$

Since the carrier escape from the GS to the WL has little effects on lasing properties [25], the $N_{G S} / \tau_{W L}^{G S}$ term in (1) and (3) can be neglected.

Based on the rate equations, the corresponding differential rate equations can be derived by considering $I, N_{W L}, N_{E S}, N_{G S}, S_{G S}$ and $g_{G S}$ as dynamic variables. In order to simplify the model and to extract the underlying physical mechanism, $f_{G S, E S}$ are assumed to be constants. To obtain the small-signal responses to a sinusoidal current modulation $w \backslash 2 \pi$ the modulation frequency, we assume solutions of the form

$$
\begin{aligned}
d N_{W L, E S, G S} & =N_{W L 1, E S 1, G S 1} e^{j w t} \\
d S_{G S} & =S_{G S 1} e^{j w t}
\end{aligned}
$$

Combining (7) into the differential rate equations, we obtain

$$
\begin{aligned}
& {\left[\begin{array}{cccc}
\gamma_{11}+j w & -\gamma_{12} & 0 & 0 \\
-\gamma_{21} & \gamma_{22}+j w & -\gamma_{23} & 0 \\
-\gamma_{31} & -\gamma_{32} & \gamma_{33}+j w & -\gamma_{34} \\
0 & 0 & -\gamma_{43} & \gamma_{44}+j w
\end{array}\right]\left[\begin{array}{c}
N_{W L 1} \\
N_{E S 1} \\
N_{G S 1} \\
S_{G S 1}
\end{array}\right]} \\
& =\frac{I_{1}}{q V_{W}}\left[\begin{array}{l}
1 \\
0 \\
0 \\
0
\end{array}\right]
\end{aligned}
$$


with

$$
\begin{aligned}
& \gamma_{11}=\frac{f_{E S}}{\tau_{E S}^{W L}}+\frac{f_{G S}}{\tau_{G S}^{W L}}+\frac{1}{\tau_{W L}^{\text {spon }}} ; \quad \gamma_{12}=\frac{1}{\tau_{W L}^{E S}} \frac{V_{D}}{V_{W}} \\
& \gamma_{21}=\frac{f_{E S}}{\tau_{E S}^{W L}} \frac{V_{W}}{V_{D}} ; \quad \gamma_{22}=\frac{f_{G S}}{\tau_{G S}^{E S}}+\frac{1}{\tau_{W L}^{E S}}+\frac{1}{\tau_{E S}^{\text {spon }}} \\
& \gamma_{23}=\frac{f_{E S}}{\tau_{E S}^{G S}} ; \quad \gamma_{31}=\frac{f_{G S}}{\tau_{G S}^{W L}} \frac{V_{W}}{V_{D}} ; \quad \gamma_{32}=\frac{f_{G S}}{\tau_{G S}^{E S}} \\
& \gamma_{33}=\frac{f_{E S}}{\tau_{E S}^{G S}}+\frac{1}{\tau_{G S}^{\text {spon }}}+v_{g} a_{G S} S_{G S} ; \quad \gamma_{34}=-v_{g} g_{G S} \\
& \gamma_{43}=\frac{\Gamma_{p} \beta_{S P}}{\tau_{G S}^{s p o n}}+\Gamma_{p} v_{g} a_{G S} S_{G S} ; \quad \gamma_{44}=-\Gamma_{p} v_{g} g_{G S}+\frac{1}{\tau_{p}} .
\end{aligned}
$$

Then, we can extract a new modulation transfer function as:

$$
H_{Q D}(w)=\frac{R_{0}}{\Delta} \equiv \frac{R_{0}}{R_{0}+j w R_{1}-w^{2} R_{2}-j w^{3} R_{3}+w^{4}}
$$

where $\Delta$ is the determinant of the matrix symbol, and the four parameters which characterize $H(w)$ are given by:

$$
\begin{aligned}
& R_{0}=w_{R}^{2} w_{R 0}^{2}-\gamma_{23} \gamma_{44}\left(\gamma_{31} \gamma_{12}+\gamma_{11} \gamma_{32}\right) \\
& R_{1}=w_{R}^{2} \Gamma_{0}+\Gamma w_{R 0}^{2}-\gamma_{23} \gamma_{32}\left(\gamma_{11}+\gamma_{44}\right)-\gamma_{31} \gamma_{12} \gamma_{23} \\
& R_{2}=w_{R}^{2}+\Gamma \Gamma_{0}+w_{R 0}^{2}-\gamma_{23} \gamma_{32} \\
& R_{3}=\Gamma+\Gamma_{0} .
\end{aligned}
$$

The relaxation resonance frequency $w_{R}$ and damping factor $\Gamma$ are approximately defined as

$$
\begin{aligned}
w_{R}^{2} & =\gamma_{33} \gamma_{44}-\gamma_{34} \gamma_{43} \\
\Gamma & =\gamma_{33}+\gamma_{44} .
\end{aligned}
$$

And the other two new parameters are:

$$
\begin{aligned}
w_{R 0}^{2} & =\gamma_{11} \gamma_{22}-\gamma_{12} \gamma_{21} \\
\Gamma_{0} & =\gamma_{11}+\gamma_{22} .
\end{aligned}
$$

Using the set of (9), equations (12) and (13) can be re-expressed as follows:

$$
\begin{aligned}
w_{R}^{2}= & \frac{v_{g} a_{G S} S_{G S}}{\tau_{p}}+\frac{\Gamma_{p} \beta_{S P} N_{G S}}{\tau_{G S}^{s p o n} S_{G S}}\left(\frac{f_{E S}}{\tau_{E S}^{G S}}+\frac{1-\beta_{S P}}{\tau_{G S}^{s p o n}}\right) \\
& +\frac{\beta_{S P}}{\tau_{G S}^{s p o n} \tau_{p}} \\
\Gamma= & v_{g} a_{G S} S_{G S}+\frac{f_{E S}}{\tau_{E S}^{G S}}+\frac{1}{\tau_{G S}^{s p o n}}+\frac{\Gamma_{p} \beta_{S P} N_{G S}}{\tau_{G S}^{s p o n} S_{G S}}
\end{aligned}
$$

where the steady-state relationship $1 / \tau_{p}-\Gamma_{p} v_{g} g_{G S}=$ $\Gamma_{p} \beta_{S P} N_{G S} /\left(\tau_{G S}^{\text {spon }} S_{G S}\right)$ has been used.

Equations (16) and (17) constitute new relations giving the resonance frequency and the damping factor for QD lasers. These equations differ from those obtained from the conventional model of QW lasers, because $w_{R}^{2}$ and $\Gamma$ contain the additional term $f_{E S} / \tau_{E S}^{G S}$, which denotes the carrier escape from the GS to the ES. Since the first term in $w_{R}^{2}$ dominates over all the other terms, the resonance frequency can be reduced to $w_{R}^{2} \approx v_{g} S_{G S} a_{G S} / \tau_{p}$. Employing this simplified definition of $w_{R}^{2}$ the damping factor can be rewritten as:

$$
\Gamma=K f_{R}^{2}+\frac{f_{E S}}{\tau_{E S}^{G S}}+\frac{1}{\tau_{G S}^{s p o n}}+\frac{\Gamma_{p} \beta_{S P} N_{G S}}{\tau_{G S}^{s p o n} S_{G S}}
$$

where the so-called K-factor is as follows:

$$
K=4 \pi^{2} \tau_{p}
$$

The expression of the K-factor is found to be the same as of the conventional one for $\mathrm{QW}$ lasers. However, the damping factor $\Gamma$ in Eq. (18) also contains the term $f_{E S} / \tau_{E S}^{G S}$ which is comparable to $K f_{R}^{2}$ even at high powers, so the offset can not be neglected.

In order to identify the influences of the WL and the ES, small contribution terms in (11) can be eliminated, then, these equations are simplified as:

$$
\begin{aligned}
& R_{0} \approx w_{R}^{2} w_{R 0}^{2} ; \quad R_{1} \approx w_{R}^{2} \Gamma_{0}+\Gamma w_{R 0}^{2} \\
& R_{2} \approx w_{R}^{2}+\Gamma \Gamma_{0}+w_{R 0}^{2} ; \quad R_{3}=\Gamma+\Gamma_{0} .
\end{aligned}
$$

Based on these expressions, the modulation transfer function (10) can be rewritten as

$$
H_{a p p}(w) \approx\left(\frac{w_{R}^{2}}{w_{R}^{2}-w^{2}+j w \Gamma}\right)\left(\frac{w_{R 0}^{2}}{w_{R 0}^{2}-w^{2}+j w \Gamma_{0}}\right) .
$$

This expression reveals that $w_{R 0}^{2}$ and $\Gamma_{0}$ play the same role in the modulation response as the resonance frequency $w_{R}^{2}$ and the damping factor $\Gamma$, respectively. It is important to analyze the effects of $w_{R 0}^{2}$ and $\Gamma_{0}$, as well as the underlying physical mechanism. The results will be discussed in the following section.

Then, the modulation bandwidth $f_{3 d B}$ can be obtained by solving $\left|H_{Q D}(w)\right|^{2}=1 / 2$

$$
2 R_{0}^{2}=\left(w^{4}-R_{2} w^{2}+R_{0}\right)^{2}+\left(R_{3} w^{3}-R_{1} w\right)^{2}
$$

and the maximum possible bandwidth $\left.f_{3 d B}\right|_{\max }$ occurs when $\Gamma^{2}=2 w_{R}^{2}$ so the value can be extracted by:

$$
2 w_{R}^{4} w_{R 0}^{4}=\left[w^{4}+w_{R}^{4}\right]\left[\left(w^{2}-w_{R 0}^{2}\right)^{2}+\left(w \Gamma_{0}\right)^{2}\right] .
$$

\section{RESUlts AND Discussion}

\section{A. Numerical Results}

All the material and QD laser parameters used in our calculations are summarized in Table I.

The capture time $\tau_{E S}^{W L}$ and relaxation time $\tau_{G S}^{E S}$ are fixed from time resolved photoluminescence experiment [27]. The direct carrier capture time from the WL to the GS $\tau_{G S}^{W L}$ is observed to be larger than the capture time $\tau_{E S}^{W L}$ in the low excitation regime [28], while under strong excitation $\tau_{G S}^{W L}$ becomes approximately the same as $\tau_{E S}^{W L}\left(\tau_{G S}^{W L} \approx \tau_{E S}^{W L}\right)$ [27]. In the calculation, the direct carrier capture time is set to $\tau_{G S}^{W L}=1.5 \tau_{E S}^{W L}$. The differential gain value is a $G S=0.5 \times$ $10^{-14} \mathrm{~cm}^{2}$.

In order to validate the model, the steady-state properties of the system are at first studied by numerically solving the four rate equations (1)-(4). The results depicted in Fig. 2 show 
TABLE I

Material PARAmeters ANd LASER PARAMETERS

\begin{tabular}{ccc}
\hline \hline Symbols & Simulation parameters & Values \\
$\mathrm{E}_{W L}$ & WL energy & $0.97 \mathrm{eV}$ \\
$\mathrm{E}_{E S}$ & ES energy & $0.87 \mathrm{eV}$ \\
$\mathrm{E}_{G S}$ & GS energy & $0.82 \mathrm{eV}$ \\
$\tau_{E S}^{W L}$ & Capture time from WL to ES & $25.1 \mathrm{ps}$ \\
$\tau_{G S}^{E S}$ & Relaxation time from ES to GS & $11.6 \mathrm{ps}$ \\
$\tau_{W L}^{\text {spon }}$ & Spontaneous time of WL & $500 \mathrm{ps}$ \\
$\tau_{E S}^{\text {spon }}$ & Spontaneous time of ES & $500 \mathrm{ps}$ \\
$\tau_{G S}^{\text {spon }}$ & Spontaneous time of GS & $1200 \mathrm{ps}$ \\
$\mathrm{n}_{r}$ & Refractive index & 3.27 \\
$\mathrm{~L}$ & Active region length & $0.11 \mathrm{~cm}$ \\
$\mathrm{~W}$ & Active region width & $3 \times 10^{-4} \mathrm{~cm}$ \\
$\mathrm{~N}$ & Number of QD layers & 5 \\
$\mathrm{~N}_{D}$ & QD density & $5 \times 10^{10} \mathrm{~cm}$ \\
$\Gamma_{p}$ & Optical confinement factor & 0.06 \\
$\beta_{S P}$ & Spontaneous emission factor: & $1 \times 10^{-4}$ \\
$\alpha_{i}$ & Internal modal loss & $6 \mathrm{~cm}^{-1}$ \\
$\mathrm{R}_{1}=\mathrm{R}_{2}$ & Mirror reflectivity & 0.3 \\
\hline \hline
\end{tabular}

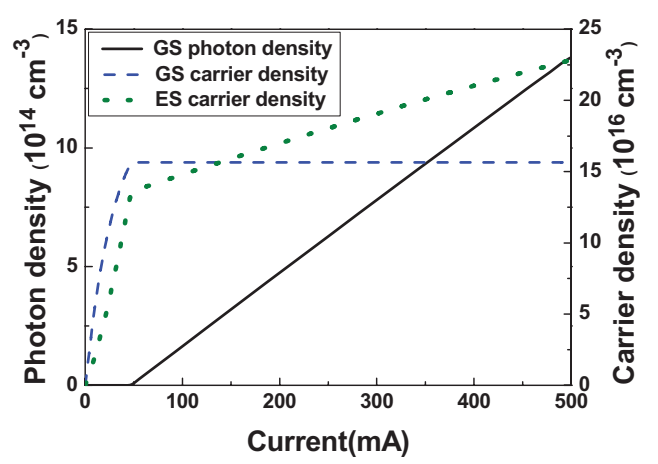

Fig. 2. Photon and carrier densities versus injected current.

that both the GS and the ES carrier populations increase with the injected current. Then, for an injected current larger than $48 \mathrm{~mA}$, the GS population are clamped which leads to the occurrence of the GS lasing emission while the ES population continues to increase with a reduced slope efficiency.

Fig. 3 illustrates the turn-on delay properties for various injected currents. With the increase of the pump current, the delay time becomes shorter which means that the carrier lifetime is decreased [29]. Both the oscillation frequency and the damping factor increase with the current. The oscillation frequencies at $1.5 \mathrm{I}_{t h}, 2 \mathrm{I}_{t h}$ and $4 \mathrm{I}_{t h}$ are $1.94 \mathrm{GHz}, 2.64 \mathrm{GHz}$ and $3.52 \mathrm{GHz}$, respectively.

Based on the steady-state results as well as on the transient response results, the modulation responses are calculated from the analytical expression (10) for various current levels and depicted in Fig. 4. Numerical results show that the relaxation frequency and the damping factor increase with the injected current. At an injected current of around $2.9 \mathrm{I}_{t h}$, the modulation bandwidth reaches the maximum value $\sim 5.5 \mathrm{GHz}$, which is in good agreement with the commonly measured values [20]-[32].

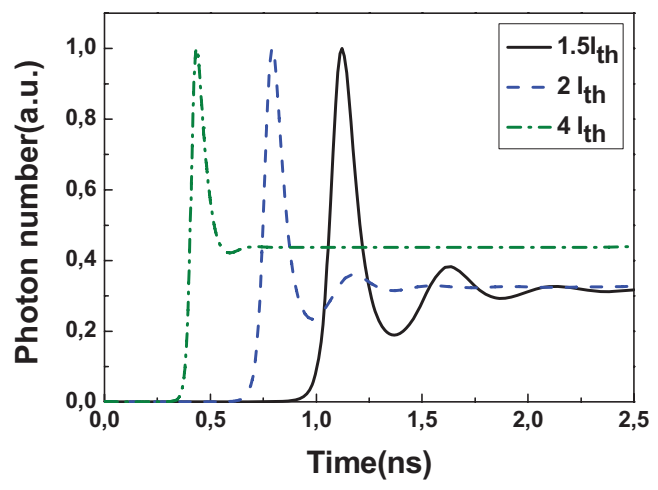

Fig. 3. Turn-on delay properties at various injected currents.

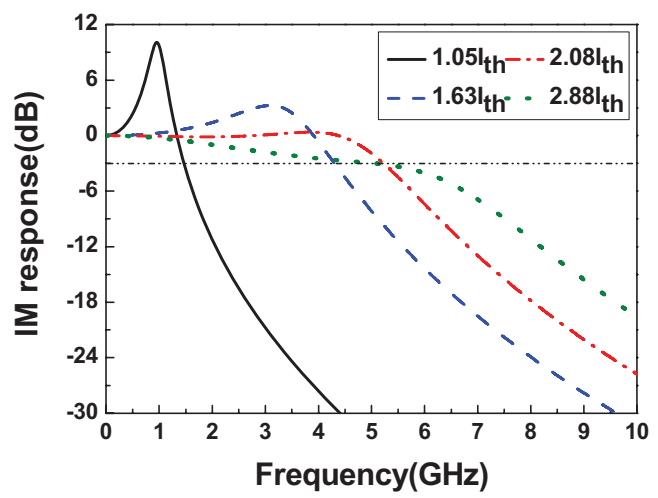

Fig. 4. Modulation responses at several injected currents, which are calculated from the new analytical expression (10).

According to (21), the modulation response $20 \log \left|H_{\text {app }}(w)\right|$ can be divided into

$$
20 \log \left|H_{\text {app }}(w)\right|=20 \log \left|H_{1}(w)\right|+20 \log \left|H_{0}(w)\right|
$$

where

$$
\begin{aligned}
& H_{1}(w)=\frac{w_{R}^{2}}{w_{R}^{2}-w^{2}+j w \Gamma} \\
& H_{0}(w)=\frac{w_{R 0}^{2}}{w_{R 0}^{2}-w^{2}+j w \Gamma_{0}} .
\end{aligned}
$$

The analytical approximation of modulation response at $2.08 \mathrm{I}_{t h}$ calculated from (24) is shown in Fig. 5 (solid line), and is compared with the exact solution given by (10). Both are found in good agreement except around the resonance peak for which the approximated value is slightly smaller than the exact solution. The behavior of $H_{1}(w)$ to the IM response (dash-dot line) is similar to that of QW lasers, and the characteristics of $w_{R}^{2}$ and $\Gamma$ characterizing $H_{1}(w)$ will be discussed in the following section. Since the modulation bandwidth of $H_{0}(w)$ is much smaller than that of $H_{1}(w)$, the total modulation bandwidth of the QD laser is limited by $H_{0}(w)$. According to the expression of $w_{R 0}^{2}$ in $H_{0}(w)$ associated with (9), the results point out that the finite carrier capture time $\tau_{E S}^{W L}$ and $\tau_{G S}^{W L}$, finite carrier relaxation time $\tau_{G S}^{E S}$ as well as Pauli blocking factor $f_{E S}$ and $f_{G S}$ are the underlying physical limitations for the enhancement of modulation bandwidth. In order to further analyze the characteristics of $f_{R 0}^{2}$ and $\Gamma_{0}$, their evolutions are 


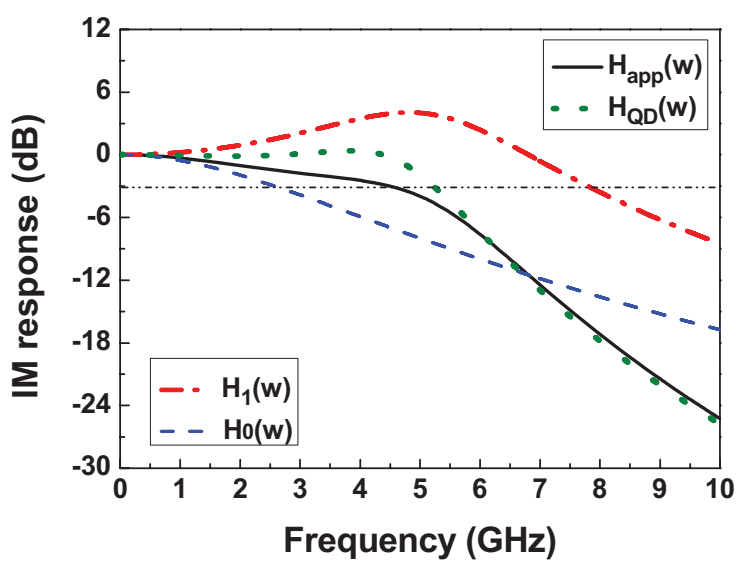

Fig. 5. Comparisons of analytical approximation (solid) calculated from (24) for modulation response and the precise value (dot) calculated from (10). Dashed line indicates contribution of $H_{0}(w)$ to the total modulation response, and dashed dotted line is contribution of $H_{1}(w)$.

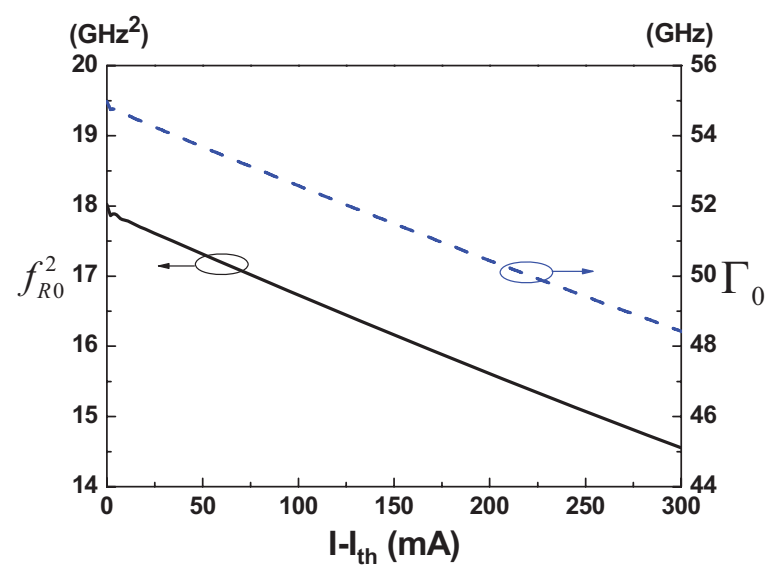

Fig. 6. Evolutions of $f_{R 0}^{2}$ and $\Gamma_{0}$ as a function of the current (I-Ith).

plotted in Fig. 6 as a function of the current $\left(\mathrm{I}-\mathrm{I}_{t h}\right)$. Inversely to the behaviors of $f_{R}^{2}$ and $\Gamma$, both $f_{R 0}^{2}$ and $\Gamma_{0}$ decrease linearly with the increased current. This is attributed to the reduced Pauli blocking factor $f_{E S}$. The relation of the two parameters is fitted as $\Gamma_{0}=1.9 f_{R 0}^{2}+20.8(\mathrm{GHz})$, and the maximum $f_{R 0}$ at threshold is only $4.2 \mathrm{GHz}$ associated with a large $\Gamma_{0}$ value of $55.0 \mathrm{GHz}$, which result in the small bandwidth of $H_{0}(w)$.

\section{B. Comparison to Experimental Results}

The device under study is an $\operatorname{InAs} / \operatorname{InP}(311 \mathrm{~B})$ QD laser [24], where the heterostructure is grown by molecular beam epitaxy (MBE) on a (311)B oriented InP substrate. The active region consists of 5 QD layers, and the measured QD density is $\sim 10^{11} \mathrm{~cm}^{2}$ [33]. The length and width of the ridge wave-guide laser are $1.1 \mathrm{~mm}$ and $3 \times 10^{-3} \mathrm{~mm}$, respectively. The laser's facets are as-cleaved. The experiment shows that the GS lasing peaks at $1.52 \mu \mathrm{m}$ at room temperature under continuous wave (cw) operation. The photon lifetime is measured to be about $5.8 \mathrm{ps}$. In this section, we use the new analytical transfer function (10) to simulate the laser modulation performance. In the calculations, the differential gain $a_{G S}$ is the only one

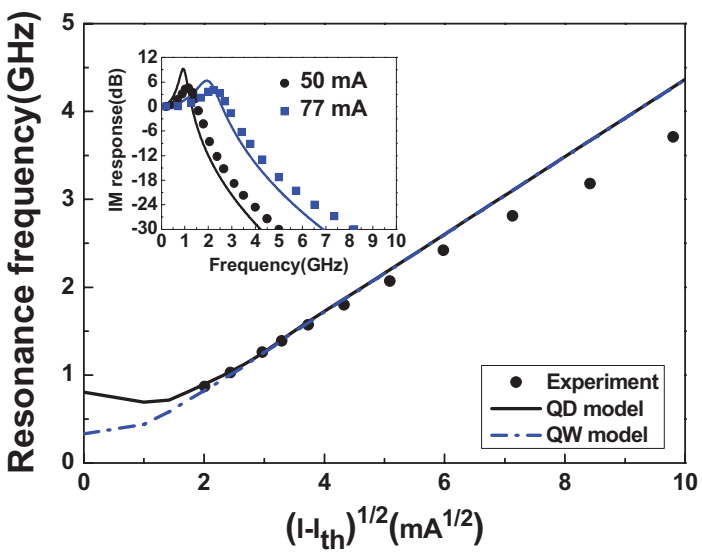

Fig. 7. Resonance frequency as a function of the current $(\mathrm{I}-\mathrm{Ith})^{1 / 2}$ and intensity modulation response at 50 and $77 \mathrm{~mA}$ (inset). Dots denote experimental results. Solid lines are theoretical results from QD model, compared with those from QW model (dashed dotted lines).

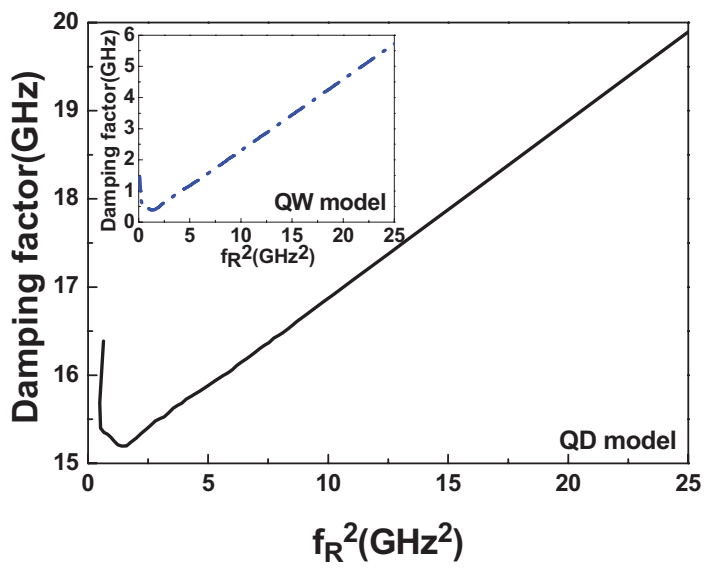

Fig. 8. Comparisons of damping factors calculated from the QD model and the QW model.

fitting parameter, which is adjusted to $0.25 \times 10^{-14} \mathrm{~cm}^{2}$. All other parameters are set to the experimental values.

Fig. 7 depicts the resonance frequency $f_{R}$ as a function of the current $\left(I-I_{t h}\right)^{1 / 2}$, while the inset shows the modulation response at two different pump currents $(50 \mathrm{~mA}$ and $77 \mathrm{~mA})$. Theoretical results (solid lines) obtained from the QD model lead to a relative good agreement with the experimental ones (dot lines). However, at large current injections, the calculated resonance frequency (lines) is found to be higher than the experimental one. Such a discrepancy is attributed to the gain compression which is not considered in the model. Analytical calculations also point out that the carrier escape from the GS to the ES induces a non-zero resonance frequency around $1 \mathrm{GHz}$ at low bias powers. Based on (16), the larger $\tau_{G S}^{E S}$ the smaller the frequency offset at threshold. This resonance frequency offset is larger than the one due to spontaneous emission only in QW lasers (dash-dot line). In the inset, the theoretical modulation responses (solid lines) match relatively well with the experimental results (dots).

Fig. 8 shows the evolution of damping factor $\Gamma$ as a function of the resonance frequency $f_{R}^{2}$. According to (18), their relationship can be fitted as $\Gamma=0.20 f_{R}^{2}+14.9(\mathrm{GHz})$, 
in comparison with the result from the conventional QW model (inset) which is $\Gamma=0.23 f_{R}^{2}+0.066(\mathrm{GHz})$. The two $\mathrm{K}$-factors are nearly the same $\sim 0.2 \mathrm{~ns}$, which is smaller than the experimental value (0.6 ns) [24]. Qualitatively, such a discrepancy can be partly attributed to the fact that the simulation does not take into account the gain saturation effects, which can be comparable to the one related to the differential gain $a_{G S}$ [12]. Besides, parasitic RC and carrier transport effects as well as the temperature effect also contribute to the discrepancy. The offset occurring in QD model is found to be much larger than that of QW model, confirming the strong damping in QD lasers. Let us stress that such a strong damping has also been pointed out to explain the QD laser's insensitivity to external perturbations [14], [18]. According to (17), carrier escape from the GS to the $\mathrm{ES}\left(f_{E S} / \tau_{E S}^{G S}\right)$ is responsible for this large damping factor. The deviation from linearity at low relaxation resonance frequency is attributed to the spontaneous emission term $\left(\Gamma_{p} \beta_{S P} N_{G S}\right) /\left(\tau_{G S}^{s p o n} S_{G S}\right)$ in the damping factor expression. This phenomenon has been observed in InGaAsP bulk lasers via a parasitic-free optical modulation technique [34].

\section{CONCLUSION}

Based on a set of four rate equations, a new analytical modulation transfer function of QD lasers has been introduced via a small-signal analysis. This numerical study clarifies the roles of the WL and of the ES to the modulation response: finite carrier capture time, finite carrier relaxation time and Pauli blocking have been found to be physical limitations to the enhancement of the modulation bandwidth. The model has been used to recast the the definitions of the resonance frequency and of the damping factor. Calculations show that carrier escape from the GS to the ES gives rise to a non-zero resonance frequency at low bias powers as well as to a strong damping factor. These results are of prime importance for further improvements of QD laser dynamic properties. Further studies will improve the rate equation model such as including the gain compression effect, the ES lasing, and treating Pauli blocking factors as variables in the differential equations.

\section{REFERENCES}

[1] G. T. Liu, A. Stintz, H. Li, K. J. Malloy, and L. F. Lester, "Extremely low room-temperature threshold current density diode lasers using InAs dots in In Ga As quantum well," Electron. Lett., vol. 35, no. 14, pp. 1163-1165, 1999.

[2] S. S. Mikhrin, A. R. Kovsh, I. L. Krestnikov, A. V. Kozhukhov, D. A. Livshits, N. N. Ledentsov, Y. M. Shernyakov, I. I. Novikov, M. V. Maximov, V. M. Ustinov, and Z. I. Alferov, "High power temperatureinsensitive $1.3 \mu \mathrm{m}$ InAs/InGaAs/GaAs quantum dot lasers," Semicond. Sci. Technol., vol. 20, no. 5, pp. 340-342, 2005.

[3] Z. Mi, P. Bhattacharya, and S. Fathpour, "High-speed $1.3 \mu \mathrm{m}$ tunnel injection quantum-dot lasers," Appl. Phys. Lett., vol. 86, no. 15, pp. 153109-1-153109-3, 2005.

[4] M. Kuntz, G. Fiol, M. Lammlin, C. Schubert, A. R. Kovsh, A. Jacob, A. Umbach, and D. Bimberg, "10Gb/s data modulation using $1.3 \mu \mathrm{m}$ InGaAs quantum dot lasers," Electron. Lett., vol. 41, pp. 244-245, Sep. 2005.

[5] H. Saito, K. Nishi, A. Kamei, and S. Sugou, "Low chirp observed in directly modulated quantum dot lasers," IEEE Photon. Technol. Lett., vol. 12 , no. 10 , pp. $1298-1300$, Oct. 2000.
[6] S. Ghosh, S. Pradhan, and P. Bhattacharya, "Dynamic characteristics of high-speed $\operatorname{In}_{0.4} \mathrm{Ga}_{0.6} \mathrm{As} / \mathrm{GaAs}$ self-organized quantum dot lasers at room temperature," Appl. Phys. Lett., vol. 81, no. 16, pp. 3055-3057, 2002.

[7] F. Grillot, B. Dagens, J. G. Provost, H. Su, and L. F. Lester, "Gain compression and above-threshold linewidth enhancement factor in $1.3 \mu \mathrm{m}$ InAs-GaAs quantum dot lasers," IEEE J. Quantum Electron., vol. 44, no. 10, pp. 946-951, Oct. 2008.

[8] N. A. Naderi, M. Pochet, F. Grillot, V. Kovanis, N. B. Terry, and L. F. Lester, "Modeling the injection-locked behavior of a quantum dash semiconductor laser," IEEE J. Sel. Topics Quantum Electron., vol. 15, no. 3, pp. 563-571, May-Jun. 2009.

[9] P. Bhattacharya, S. Ghosh, S. Pradhan, J. Singh, Z. K. Wu, J. Urayama, K. Kyoungsik, and T. B. Norris, "Carrier dynamics and high-speed modulation properties of tunnel injection InGaAs-GaAs quantum-dot lasers," IEEE J. Quantum Electron., vol. 39, no. 8, pp. 952-962, Aug. 2003.

[10] R. R. Alexander, D. T. D. Childs, H. Agarawal, K. M. Groom, H. Y. Liu, M. Hopkinson, R. A. Hogg, M. Ishida, T. Yamamoto, M. Sugawara, Y. Arakawa, T. J. Badcock, R. J. Royce, and D. J. Mowbray, "systematic study of the effects of modulation p-doping on $1.3-\mu \mathrm{m}$ quantum-dot lasers," IEEE J. Quantum Electron., vol. 43, no. 12, pp. 1129-1139, Dec. 2007.

[11] A. Fiore and A. Markus, "Differential gain and gain compression in quantum-dot lasers," IEEE J. Quantum Electron., vol. 43, no. 3, pp. 287-294, Apr. 2007.

[12] L. A. Coldren and S. W. Corzine, Diode Lasers and Photonic Integrated Circuits. New York: Wiley, 1995.

[13] M. Kuntz, N. N. Ledentsov, D. Bimberg, A. R. Kovsh, and V. M. Ustinov, "Spectrotemporal response of $1.3 \mu \mathrm{m}$ quantum-dot lasers," Appl. Phys. Lett., vol. 81, no. 20, pp. 3846-3848, 2002.

[14] D. O'Brien, S. P. Hegarty, G. Huyet, J. G. McInerney, T. Laemmlin, D. Bimberg, V. M. Ustinov, A. E. Zhukov, A. R. Mikhrin, and A. R. Kovsh, "Feedback sensitivity of $1.3 \mu \mathrm{m}$ InAs/GaAs quantum dot laser," Electron. Lett., vol. 39, no. 25, pp. 1819-1820, 2003.

[15] E. Malic, K. J. Ahn, M. J. Bormann, P. Hovel, E. Scholl, A. Knorr, M. Kuntz, and D. Bimberg, "Theory of relaxation oscillations in semiconductor quantum dot lasers," Appl. Phys. Lett., vol. 89, no. 10, pp. 101107-1-101107-3, 2006.

[16] D. Klotzkin, K. Kamath, and P. Bhattacharya, "Quantum capture times at room temperature in high-speed InGa As-GaAs self-organized quantum-dot lasers," IEEE Photon. Technol. Lett., vol. 9, no. 10, pp. 1301-1303, Oct. 1997.

[17] M. Ishida, N. Hatori, T. Akiyama, K. Otsubo, Y. Nakata, H. Ebe, M. Sugawara, and Y. Arakawa, "Photon lifetime dependence of modulation efficiency and $\mathrm{K}$ factor in $1.3 \mu \mathrm{m}$ self-assembled InAs/GaAs quantum-dot lasers: Impact of capture time and maximum modal gain on modulation bandwidth," Appl. Phys. Lett., vol. 85, no. 18, pp. 4145-4147, 2004.

[18] D. O'Brien, S. P. Hegarty, G. Huyet, and A. V. Uskov, "Sensitivity of quantum dot semiconductor lasers to optical feedback," Opt. Lett., vol. 29, no. 10, pp. 1072-1074, 2004.

[19] L. Asryan, Y. Wu, and R. Suris, "Carrier capture delay and modulation bandwidth in an edge-emitting quantum dot laser," Appl. Phys. Lett., vol. 98, no. 13, pp. 131108-1-131108-3, 2011.

[20] M. Sugawara, Self-Assembled InGaAs-GaAs Quatum Dots. New York: Academic, 1999.

[21] D. Deppe and D. Huffaker, "Quantum dimensionality, entropy, and the modulation response of quantum dot lasers," Appl. Phys. Lett., vol. 77, no. 21 , pp. $3325-3327,2000$

[22] O. Qasaimeh and H. Khanfar, "High-speed characteristics of tunnelling injection and excited-state emitting InAs/GaAs quantum dot lasers," IEE Proc. Optoelectron., vol. 151, no. 3, pp. 143-150, 2004.

[23] M. Gioannini, A. Sevega, and I. Montrosset, "Simulations of differnetial gain and linewidth enhancement factor of quantum dot semicondutor lasers," Opt. Quantum Electron., vol. 38, nos. 4-6, pp. 381-394, 2006.

[24] A. Martinez, K. Merghem, S. Bouchoule, G. Moreau, A. Ramdane, J. G. Provost, F. Alexandre, F. Grillot, O. Dehaese, R. Piron, and S. Loualiche, "Dynamic properties of $\operatorname{InAs} / \operatorname{InP}(311 \mathrm{~B})$ quantum dot Fabry-Perot lasers emitting at 1.52- $\mu \mathrm{m}$," Appl. Phys. Lett., vol. 93, no. 2, pp. 021101-1-021101-3, 2008.

[25] K. Veselinov, F. Grillot, C. Cornet, J. Even, A. Bekiarski, M. Gioannini, and S. Loualiche, "Analysis of the double laser emission occurring in 1.55- $\mu \mathrm{m}$ InAs-InP (113)B quantum-dot lasers," IEEE J. Quantum Electron., vol. 43, no. 9, pp. 810-816, Sep. 2007. 
[26] F. Grillot, K. Veselinov, M. Gioannini, I. Montrosset, J. Even, R. Piron, E. Homeyer, and S. Loualiche, "Spectral analysis of $1.55 \mu \mathrm{m}$ InAs-InP(113)B quantum-dot lasers based on a multipopulation rate equations model," IEEE J. Quantum Electron., vol. 45, no. 7, pp. 872-878, Jul. 2009.

[27] P. Miska, J. Even, O. Dehaese, and X. Marie, "Carrier relaxation dynamics in InAs/InP quantum dots," Appl. Phys. Lett, vol. 92, no. 19, pp. 191103-1-191103-3, 2008.

[28] U. Bockelmann and T. Egeler, "Electron relaxation in quantum dots by means of Auger processes," Phys. Rev. B Condens. Phys., vol. 46, no. 23, pp. 15574-15577, 1992.

[29] G. P. Agrawal and N. K. Dutta, Long Wavelength Semiconductor Lasers. New York: Academic, 1986.

[30] M. Sugawara, N. Hatori, M. Ishida, H. Ebe, and Y. Arakawa, "Recent progress in self-assembled quantum-dot optical devices for optical telecommunication: Temperature-insensitive $10 \mathrm{~Gb} / \mathrm{s}-1$ directly modulated lasers and $40 \mathrm{~Gb} / \mathrm{s}-1$ signal-regenerative amplifiers," J. Phys. D Appl. Phys., vol. 38, no. 13, pp. 2126-2134, 2005.

[31] P. Bhattacharya and Z. Mi, "Quantum-dot optoelectronic devices," Proc. IEEE, vol. 95, no. 9, pp. 1723-1740, Sep. 2007.

[32] R. Krebs, F. Klopf, S. Rennon, J. P. Reithmaier, and A. Forchel, "High frequency characteristics of InAs/ GalnAs quantum dot distributed feedback lasers emitting at $1.3 \mu \mathrm{m}$," Electron. Lett., vol. 37, no. 20, pp. 1223-1225, 2001

[33] N. F. Massé, E. Homeyer, A. R. Adams, S. J. Sweeney, O. R. Piron, F. Grillot, and S. Loualiche, "Temperature and pressure dependence of the recombination processes in $1.5 \mu \mathrm{m}$ InAs/InP (311)B quantum dot lasers," Appl. Phys. Lett., vol. 91, no. 13, pp. 131113-131115, 2007.

[34] C. B. Su, J. Eom, C. William, C. Rideout, H. Lange, C. B. Kim, R. B. Lauer, and J. S. Lacourse, "Characterization of the dvnamics of semiconductor lasers using optical modulation," IEEE J. Quantum Electron., vol. 28, no. 1, pp. 118-127, Jan. 1992.

[35] K. Ludge, E. Scholl, E. Viktorov, and T. Erneux, "Analytical approach to modulation properties of quantum dot lasers," J. Appl. Phys., vol. 109, no. 10, p. 103112, 2011.

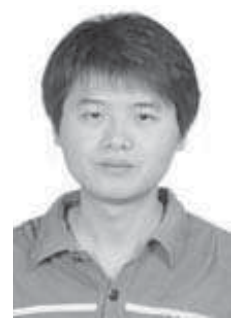

Cheng Wang was born in Tengzhou, China, in 1987. He received the M.S. degree in physical electronics from the Harbin Institute of Technology, Harbin, China, in 2011. He is currently pursuing the Ph.D degree with the Department of Materials and Nanotechnology, National Institute of Applied Sciences, Rennes, France.

His current research interests include modeling quantum dot materials and lasers.

Mr. Wang is Student Member of the SPIE.

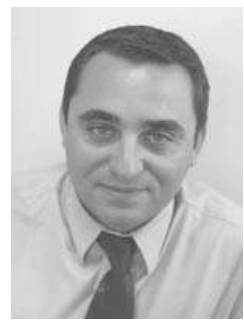

Frédéric Grillot (SM'12) was born in Versailles, France, on August 22, 1974. He received the M.Sc. degree from the University of Dijon, France in 1999, the $\mathrm{PhD}$ degree from the University of Besançon, France, in 2003 and the Research Habilitation from the University of Paris VII, France, in 2012. His doctoral research activities were conducted with the Optical Component Research Department, AlcatelLucent, involving in research on the effects of the optical feedback in semiconductor lasers, and the impact this phenomenon has on optical communi-

cation systems.

He was with the Institut d'Electronique Fondamentale, University ParisSud, Orsay, France, from 2003 to 2004, where he was engaged in research on integrated optics modeling and on Si-based passive devices for optical interconnects. Since September 2004, he has been with the Institut National des Sciences Appliquées, Rennes, France, as an Associate Professor. From 2008 to 2009, he was a Visiting Professor with the University of New-Mexico, Albuquerque, involved in research on optoelectronics with the Center for High Technology Materials. Since September 2011, he has been an Adjunct Associate Professor with the Communications and Electronic Department, Telecom Paristech, Paris, France. He is a regular reviewer for several highimpact factor international journals. He is the author or co-author of 37 journal papers, one book, two book chapters, and more than 70 contributions in international conferences. His current research interests include advanced lase diodes using new materials like quantum dots for low-cost applications and nonlinear dynamics in semiconductor lasers.

Dr. Grillot is a Senior Member of the SPIE. He is a member of the Optical Society of America and the ESA.

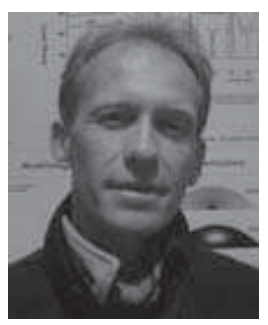

Jacky Even was born in Rennes, France, in 1964. $\mathrm{He}$ received the $\mathrm{Ph} . \mathrm{D}$. degree from the University of Paris VI, Paris, France, in 1992.

He was a Research and Teaching Assistant with the University of Rennes I, Rennes, France, from 1992 to 1999. He has been a Full Professor of optoelectronics with the Institut National des Sciences Appliquées (INSA), Rennes, since 1999. He was the Head of the Materials and Nanotechnology Department, INSA, from 2006 to 2009 . He is currently the Director of Education, INSA. He is the author or coauthor of 120 papers and 125 contributions in international conferences. His current research interests include theoretical study of the electronic, optical, and nonlinear properties of quantum well and quantum dot structures, and the simulation of optoelectronic devices. 